\title{
On the nature of "collisionless" Landau damping
}

\author{
R. Bilato ${ }^{a}$ and M. Brambilla ${ }^{a}$ \\ ${ }^{\mathrm{a}}$ Max-Planck Intitut für Plasmaphysik - D-85748 Garching, Germany
}

\begin{abstract}
The essential role of collisions in Landau damping is illustrated with heuristic but quantitatively accurate arguments. They show that above a critical (and very weak) collisionality the linearization of the Vlasov equation for the perturbation and the quasilinear description of the evolution of the background distribution function are simultaneously justified, and yield a closed and internally consistent model. We argue that phase mixing is not the same as irreversibility, but greatly enhances the efficiency of collisions in causing it.
\end{abstract}

Key words: Landau Damping, Linearized Vlasov Equation, Quasilinear Diffusion Coefficient, Plasmas PACS: 52.20.Dq, 52.35.Fp, 52.25.Dg, 05.10.Gg,

\section{Introduction}

Since its first derivation in 1946 [1], Landau damping has been the object of much discussion, aiming at understanding the "paradox" of an irreversible process being predicted by a collisionless model, namely Vlasov equation. Even after the experimental confirmation of the effect [2] the discussion went on, and is still far from damped away (e.g. [3] and references therein).

In this paper we address the physics of Landau damping mechanism with a very simple model which is nevertheless able to determine accurately the limits of the applicability of the Landau results. For simplicity, we confine the analysis to the steady state of externally excited waves which propagate and are absorbed in fusion plasmas [4]. The key of our argument is the observation that Vlasov equation is not an exact description of the dynamics of charged particles in the plasma, but only holds for times shorter than the collision relaxation time appropriate to the phenomena under consideration. For slower phenomena Fokker-Planck-Landau equation, must be used. In the following we describe a

Email address: Roberto.Bilato@ipp.mpg.de (R. Bilato). 
physical picture which also retains the main feature of the transition to nonlinear Landau damping $\left(\nu_{\text {coll }} \ll \nu_{\text {crit }}\right)$ on one side, and to the collisional regime typical of weakly ionized plasmas and gases $\left(\nu_{\text {coll }}>\nu\right)$ on the other. In the case of linearized Vlasov-Poisson set of equations, the role of collisions has been recently addressed by $\mathrm{Ng}$, Bhattacharjee, and Skiff [5] who found that collisions change qualitatively the eigenmodes and the spectra of the system.

\section{Model of Landau Damping}

We consider the motion of an electron in the electrostatic potential of a longitudinal wave $E(x, t)=E_{0} \cos (k x-\omega t)$. The change of velocity in the time interval $\Delta t$ is

$$
\Delta v=-\frac{e E_{0}}{m_{e}} \int_{0}^{\Delta t} \mathrm{~d} t \cos (k x(t)-\omega t)
$$

where $x(t)$ is the solution of the equation for the nonlinear harmonic oscillator in the wave. For times shorter than the bouncing time $\Delta t<t_{\text {bounc }}$ in Eq. (1) we can simply use $x\left(t_{0}+\Delta t\right) \approx x_{0}+v_{0} t[6]$ to obtain:

$$
\Delta v=2 \frac{e E_{0}}{m_{e}} \frac{1}{k v_{0}-\omega} \sin \left(\frac{k v_{0}-\omega}{2} \Delta t\right) \cos \left(k x_{0}+\frac{k v_{0}-\omega}{2} \Delta t\right)
$$

The amount of power exchanged between resonant particles and waves is proportional to $\Delta v \Delta v$, averaged over the initial position $x_{0}$ (assumed random):

$$
\begin{aligned}
<\Delta v \Delta v>_{\Delta t} & \equiv \frac{1}{2 \pi} \frac{1}{\Delta t} \int_{0}^{2 \pi} \mathrm{d}\left(k x_{0}\right) \Delta v \Delta v \\
& =2\left(\frac{e E_{0}}{m_{e}}\right)^{2} \frac{1}{(k v-\omega)^{2} \Delta t} \sin ^{2}\left(\frac{k v-\omega}{2} \Delta t\right) \\
& \stackrel{\Delta t \rightarrow \infty}{\longrightarrow} \pi\left(\frac{e E_{0}}{m_{e}}\right)^{2} \delta(k v-\omega)
\end{aligned}
$$

where we have omitted the subscript of the velocity. Fig. (1.a) shows as $\langle\Delta v \Delta v\rangle_{\Delta t}$ changes with $\Delta t$ : as $\Delta t$ increases the resonant particle exchange more energy per unit time (height), but it stays in resonance for a shorter time (width). Thus the total energy absorbed during $\Delta t$ does not depend on $\Delta t$.

To evaluate the energy per unit time $\mathcal{K}$ exchanged by electrons with the wave, we describe the slow evolution of the distribution function $f$ due to interaction with hfwaves as a random walk:

$$
\frac{\partial f}{\partial t}=\frac{1}{2} \frac{\partial}{\partial v}\left[\langle\Delta v \Delta v\rangle_{\Delta t} \frac{\partial f}{\partial v}\right]
$$

which gives:

$$
\frac{\partial \mathcal{K}}{\partial t}=\int \mathrm{d} v \frac{m_{e} v^{2}}{2} \frac{\partial f}{\partial t}=-\int \mathrm{d} v \frac{m_{e}}{2} v<\Delta v \Delta v>_{\Delta t} \frac{\partial f}{\partial v}
$$



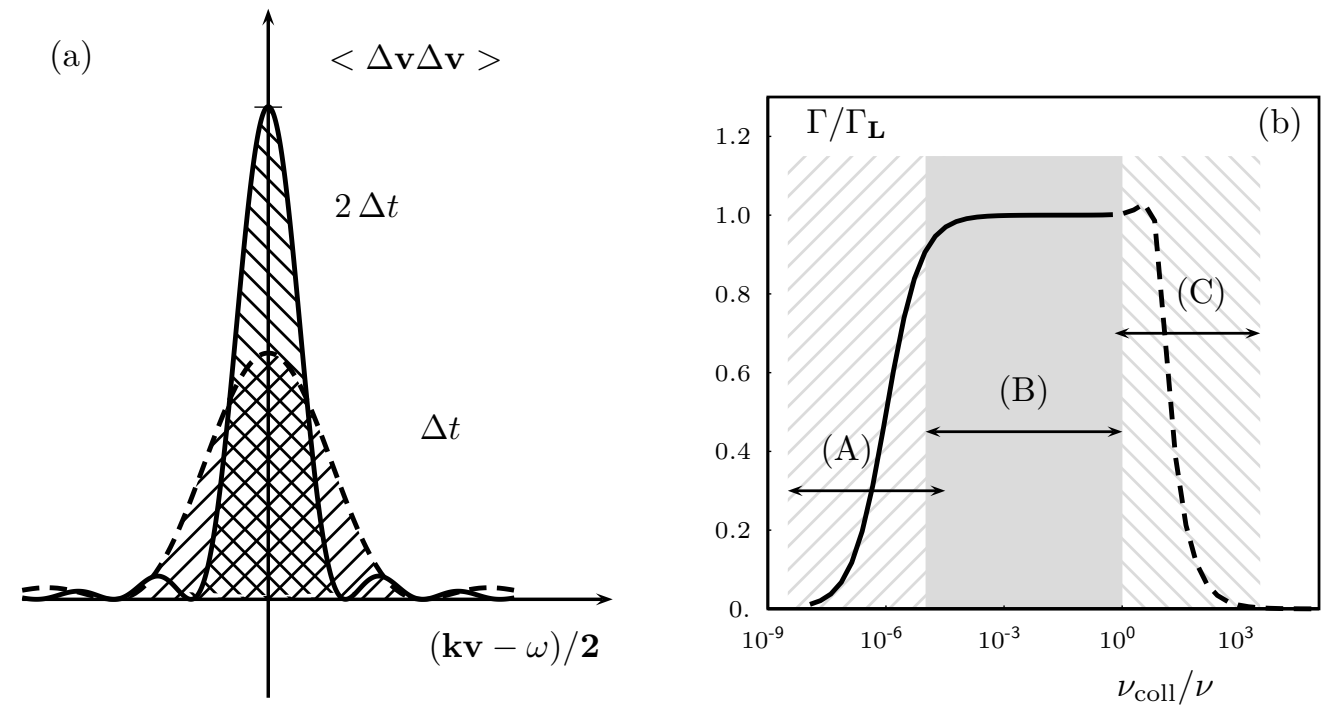

Fig. 1. (a): $\langle\Delta v \Delta v\rangle_{\Delta t}$ of Eq. (3) for two values of $\Delta t$; (b). $\Gamma_{L} / \Gamma$ as function of $\nu_{\text {coll }} / \nu$ (with $\nu=\varnothing / 2 \pi$ ), obtained using Eq. (3) in Eq. (6) and $v_{\mathrm{ph}}=2 v_{\text {the }}$, the last relevant only for $\nu_{\text {coll }}>\nu$. The regions (A) and (B) refers to the nonlinear [7] and linear Landau damping [1] regimes. For $\nu_{\text {coll }}>\nu$ the system enters in the regime typical of high collisional plasmas [8] and neutral gases [9].

In the limit $\nu_{\text {coll }} / \nu \ll 1, f$ differs from a Maxwellian $f_{M}$ mainly in the small interval $\left[v_{\mathrm{ph}}-\delta v, v_{\mathrm{ph}}+\delta v\right][7]$, with $v_{\mathrm{ph}}$ It can been easily shown $[10,11]$ that the velocity-derivative of $f$ needed in (6) can be approximated as:

$$
\left.\frac{\partial f}{\partial v}\right|_{v=v_{\mathrm{ph}}}=\left.\frac{\nu_{\mathrm{coll}}}{\nu_{\mathrm{coll}}+\nu_{\mathrm{crit}}} \frac{\partial f_{M}}{\partial v}\right|_{v=v_{\mathrm{ph}}}
$$

with

$$
\nu_{\text {crit }}=\left.\left(\frac{v_{\text {ph }}}{v_{\text {the }}}\right)^{3} \frac{2 \delta v}{v_{\text {the }}^{2}} \frac{\mathrm{d} v}{\mathrm{~d} t}\right|_{v=v_{\mathrm{ph}}}=\varnothing\left(\frac{v_{\mathrm{ph}}}{v_{\text {the }}}\right)^{2}\left(\frac{e \Phi_{0}}{m_{e} v_{\text {the }}^{2} / 2}\right)^{3 / 2} \equiv \nu_{\text {crit }}
$$

with $E_{0}=k \Phi_{0}$ and $v_{\mathrm{ph}}=\omega / k$. Upon using Eq. (3) and Eq. (7) in Eq. (6), the heating rate as function of the normalized collision frequency $\nu_{\text {coll }} / \nu$ is reported as solid line in Fig. (1.b). For $\nu_{\text {coll }} \ll \nu$, we can approximate:

$$
\Gamma=\frac{\nu_{\text {coll }}}{\nu_{\text {coll }}+\nu_{\text {crit }}} \Gamma_{L}
$$

with $\Gamma_{L}$ the damping rate at the plateau $\nu_{\text {crit }} \ll \nu_{\text {coll }} \ll \nu$,

$$
\Gamma_{L}=\frac{\partial \mathcal{K}}{\partial t}=\frac{\pi}{2} \frac{e^{2} E_{0}^{2}}{m_{e}}\left(-\frac{\partial f_{M}}{\partial v}\right)_{v_{\mathrm{ph}}}
$$

equal to the rate evaluated by Landau (see Sec. 8.2 of [6] for details). 
Equation (9) describes two collisional regimes [10]:

$$
\begin{aligned}
& \text { (A) : } \nu_{\text {coll }} \ll \nu_{\text {crit }} \quad \text { with } \Gamma=\left(\nu_{\text {coll }} / \nu_{\text {crit }}\right) \Gamma_{L} \\
& \text { (B) : } \nu_{\text {crit }} \ll \nu_{\text {coll }} \ll \nu \text { with } \Gamma=\Gamma_{L}
\end{aligned}
$$

Regime (A) is the low-collisionality, or large amplitude, regime studied by Zakharov and Karpman [7], in which the absorption is proportional to the collision rate, and depends explicitly on the amplitude $E_{0}$. Although in this regime the derivation [10] is, strictly speaking, oversimplified, the result Eq. (9) agrees well with the damping rate evaluated by a more rigorous model (cf. Eq.(43) in [7]). In regime (B) the familiar Landau damping rate is recovered, which does not depend on either $\nu_{\text {coll }}$ or $E_{0}$. Although usually called 'collisionless', this regime would be more appropriately called 'plateau', as in the theory of neoclassical diffusion in tokamaks [12]. Thus the classical Landau damping mechanism which predicts a damping rate independent from the collision frequency applies only for low collision frequency, but not for $\nu_{\text {coll }}$ exactly zero, as the adjective "collisionless" would suggest $[10,6]$.

For $\nu_{\text {coll }} / \nu>1$ the damping rate decreases with $\nu_{\text {coll }} / \nu$, since the duration of the waveparticle interaction is much less than the period of the wave. This is the typical regime of high collisional plasmas [8] (hardly relevant for fusion plasma) and neutral gases [9].

To understand the physical meaning of $\nu_{\text {crit }}$ we observe that the steady state solution of the Fokker-Planck equation (FPE) emerges from a balance between the trapping of resonant particles in the wave electric field and collisions. The trapping in the wave field of amplitude $E_{0}$ tends to drive the distribution function away from the thermal equilibrium in the velocity interval $\left|v-v_{\mathrm{ph}}\right|<\delta v \equiv\left(2 e E_{0} /(m k)\right)^{1 / 2}$. An initially trapped particle can be detrapped as soon as its velocity is changed of an amount $\delta v$ [4]. Because of the random walk nature of collision diffusion in velocity space, the characteristic detrapping time is:

$$
\tau_{\text {detr }}=\tau_{\text {coll }}\left(v_{\text {ph }}\right)\left(\frac{\delta v}{v_{\text {the }}}\right)^{2}=\tau_{\text {coll }}\left(\frac{v_{\text {ph }}}{v_{\text {the }}}\right)^{3} \frac{e \Phi_{0}}{\left(m v_{\text {the }}^{2}\right) / 2}
$$

The linearized Vlasov equation is justified when a particle initially trapped in the wave is detrapped in a time shorter than the bouncing time in the wave well, $\tau_{\text {bounc }}=$ $\varnothing^{-1}\left[e \Phi_{0} /\left(m v_{\mathrm{ph}}^{2} / 2\right)\right]^{-1 / 2}$. If $\nu_{\text {coll }}=\tau_{\text {coll }}^{-1} \gg \nu_{\text {crit }}$ the particle exits from resonance before feeling any nonlinearity, and carrying away the energy exchanged with the wave while in resonance. It is replaced by a new particle whose bouncing motion will have a random phase with respect to the wave (this justifies the "random phase" assumption made to write Eq. (3).

If collisionality increases, the rate of detrapping increases, but the energy carried away by each particle decreases, and vice versa. The result of this irreversible process is Landau damping. Although collisions are essential to introduce irreversibility, the resulting damping rate is independent from the collision frequency, and, in fact, from the particular nature of the dissipative mechanism. This is an universal property of resonant phenomena, resulting from the combination of a very long interaction time and a very weak dissipation mechanism, and is the reason why analogies with Landau damping are 
increasingly recognized in many fields outside plasma physics [13,14].

\section{Quasilinear Diffusion Coefficient}

That Eq. (9) correctly describes the qualitative behaviour of $\Gamma$ as a function of $\nu_{\text {coll }} / \nu_{\text {crit }}$ can be checked in a different way, which at the same time shows that in the "plateau" regime the background distribution function $f$ obeys the familiar quasilinear kinetic equation [15]. We consider the motion of individual electrons in an infinite set of electrostatic waves, all with the same electric field amplitude, and with equally spaced wavevectors $k_{n}$. However, we use this model in a regime where the single particle is effectively interacting with only one wave. As noted by Stix [6], the corresponding Hamiltonian yields the standard map [16], of which a number of analysis are available in literature [17]. Coulomb collisions can be added to the map as random kicks $\delta v_{j}$

$$
v_{j+1}=v_{j}+\epsilon \sin z_{j}+\delta v_{j+1}, \quad x_{j+1}=x_{j}+v_{j+1}
$$

sampled out of a Gaussian distribution $f(\delta v)=\left(2 \pi D_{\text {coll }}\right)^{-1 / 2} \exp \left[-(\delta v)^{2} / 2 D_{\text {coll }}\right]$ with $D_{\text {coll }}=v_{\text {the }}^{2} \nu_{\text {coll }} / 2$ the collision diffusion coefficient in velocity space. This implementation of collisions is consistent with a diffusion process only when the kicks are small and not frequent, and this is the case when $\nu_{\text {coll }} \ll \nu$. The map equations (12) are written in dimensionless variables by rescaling length and time respectively by $k$ and $\omega^{2} / 2 \pi c k$, so that $\epsilon=e \Phi_{0} k^{2} / m_{e} \omega^{2}$ is the normalized amplitude of the wave electric field. The ratio between the diffusion coefficient in phase space and its quasilinear part, $D / D_{\mathrm{QL}}$, quantifies the stochasticity degree of the system. When the phase space is dominated by regular regions (i.e. has well defined Kolmogorov-Arnold-Moser (KAM) surfaces), $D / D_{\mathrm{QL}} \ll 1$. On the other hand, when the phase space is mainly stochastic, $D / D_{\mathrm{QL}} \approx 1$.

Figure (2.a) shows results [15] for $D / D_{\mathrm{QL}}$ as function of $\epsilon$ with (solid line) and without collisions (dashed line). In the absence of collisions, $D / D_{\mathrm{QL}}$ is negligible when the electric field is below the Chirikov overlapping threshold [16], $\epsilon<\epsilon_{\mathrm{C}}=0.989$ (vertical line in Fig. (2)). For $\epsilon$ above $\epsilon_{\mathrm{C}}$ the motion becomes extremely sensitive to the initial conditions, producing a greatly increased fine-graining of the particle distribution in phase space. Soon above this threshold the finite numerical resolution has the same effect as an external noise perturbing the system, and $D / D_{\mathrm{QL}}$ rapidly approaches unity. In the presence of extrinsic diffusion induced by collisions, $D / D_{\mathrm{QL}}$ has a completely different behaviour, in particular at low amplitudes. For $\epsilon \ll \epsilon_{\mathrm{C}}$ collisions are the dominant effect, and $D / D_{\mathrm{QL}} \approx 1$; only as $\epsilon$ increases the order introduced by the wave field (KAM surfaces) reduces diffusion in phase space, and $D / D_{\mathrm{QL}}$ approaches the behaviour of the collisionless case: this is regime (A) of Eq. (9). This trend continues up and beyond the point where resonance overlapping appears.

The regime relevant to verify the correctness of Eq. (9) is the case of $\epsilon \ll \epsilon_{\mathrm{C}}$, in which the effects of resonance overlapping are completely negligible. In this limit, in the collisionless case, the large majority of particles can interact resonantly with at most one wave of the set, as assumed in the derivation of Eq. (9). Collisions, moreover, are the only source of stochasticity. Figure (2.b) shows $D / D_{\mathrm{QL}}$ (solid lines) and $\Gamma / \Gamma_{L}$ (dashed lines) as functions of $D_{\text {coll }}$ for three values of $\epsilon \ll 1$. The transition to the quasilinear behaviour 

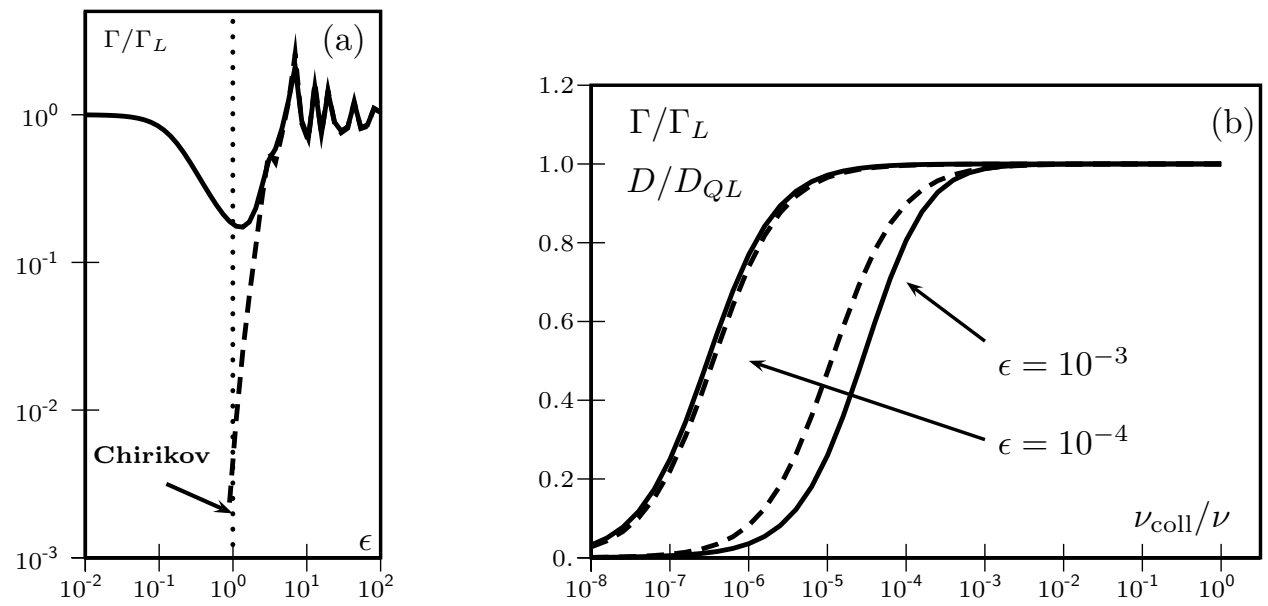

Fig. 2. (a) The diffusion coefficient as function of $\epsilon$ with collisions (solid line) and without collisions (dashed line). In the absence of collisions the diffusion coefficient vanishes for $\epsilon$ less than the Chirikov threshold [16] (vertical line). (b): Diffusion coefficient (solid line) and damping rate (dashed line) as function of the normalized collision diffusion coefficient $D_{\text {coll }}=\nu_{\text {coll }} v_{\text {the }}^{2} / 2$. Details are given in [15].

occurs at $D_{\text {crit }}=v_{\text {the }}^{2} \nu_{\text {crit }} / 2$ (in dimensionless units $D_{\text {crit }}=\epsilon^{3 / 2} / \sqrt{2}$ ); precisely at the same collisionality $\Gamma$ jumps to its plateau value.

\section{Conclusion}

The originality of the physical picture of Landau damping proposed in this note stems not from its single parts which are well-know (see for instance [6]), but in its ability to predict and explain the regimes of this important mechanism. Despite its simplicity, this approach gives results in agreement with sophisticate analytical analysis and Monte Carlo simulations $[18,15]$ (in particular Fig. (1.b) is to be compare with Fig. (2) of [18]), and, therefore, it offers a pedagogical introduction to the physics of Landau damping mechanism. It also clarifies the true role of phase mixing: fine graining in phase space is not itself an irreversible process, as shown by phenomenon of 'echos' [19], but enormously increases the efficiency of collisions in causing irreversibility. Here, again, Landau damping is a paradigm for a large variety of irreversibility paradoxes, including the most famous one, namely the observation of irreversible behaviour in closed systems. Indeed, explaining Landau damping with phase mixing is akin to equate irreversibility and ergodicity. In both cases, the paradox arises because an idealized model (collisionless Vlasov equation, closed system) is used far beyond its validity range. For times comparable with the inverse Landau damping rate the reversible Vlasov equation must be replaced by its irreversible linearized form for the perturbation, together with the quasilinear kinetic equation for the background plasma. Similarly, the irreversibility paradox disappears when it is realized that no macroscopic system which is ergodic, however well insulated, can be regarded as closed for times ever faintly comparable with its Poincaré recurrence time.

Finally, it should go without mentioning that our considerations apply without restriction only to stable plasmas. In the case of growing waves, whether or not the motion of 
resonant particles remains linear before collisional detrapping occurs, so that quasilinear saturation can be reached, depends on the growth rate, on the spectrum of unstable waves (a broad spectrum causing more fine graining in velocity space), and, particularly in numerical simulations, also on the initial amplitude of the perturbation.

Acknowledgment - We are indebted to Prof. Karl Lackner and Dr. Arthur Peeters for a number of useful discussions on this topic.

\section{References}

[1] L. D. Landau, On the vibrations of the electronic plasmas, J. Phys. (Moscow) 10 (1946) 25

[2] J. H. Malmberg, C. B. Wharton, Collisionless damping of electrostatic plasma waves, Phys. Rev. Lett. 13 (6) (1964) 184-186.

[3] D. D. Ryutov, Landau damping: half a century with the great discovery, Plasma Physics and Controlled Fusion 41 (3A) (1999) A1-A12.

URL http://stacks.iop.org/0741-3335/41/A1

[4] M. Brambilla, Kinetic Theory of Plasma Waves, Oxford University Press, USA, 1998.

[5] C. S. Ng, A. Bhattacharjee, F. Skiff, Kinetic eigenmodes and discrete spectrum of plasma oscillations in a weakly collisional plasma, Phys. Rev. Lett. 83 (10) (1999) 1974-1977.

[6] T. H. Stix, Waves in Plasmas, AIP Press, 1992.

[7] V. Zakharov, V. Karpman, On the nonlinear theory of the damping of plasma waves, Sov. Phys. JETP 16 (1963) 351.

[8] M. M. Turner, Collisionless electron heating in an inductively coupled discharge, Phys. Rev. Lett. 71 (12) (1993) 1844-1847.

[9] P. Stubbe, Landau damping of sound in a rarefied neutral gas, Physics of Fluids 6 (9) (1994) 31643174 .

URL http://link.aip.org/link/?PHF/6/3164/1

[10] E. Canobbio, Neoclassical theory of magnetic pumping geometry, in: IAEA (Ed.), Plasma Physics and Controlled Nuclear Fusion Research, Vol. 3, Vienna, 1971, p. 491.

[11] S. P. Auerbach, Collisional damping of langmuir waves in the collisionless limit, Physics of Fluids 20 (11) (1977) 1836-1844. URL http://link.aip.org/link/?PFL/20/1836/1

[12] F. L. Hinton, R. D. Hazeltine, Theory of plasma transport in toroidal confinement systems, Rev. Mod. Phys. 48 (2) (1976) 239-308.

[13] S. H. Strogatz, R. E. Mirollo, P. C. Matthews, Coupled nonlinear oscillators below the synchronization threshold: Relaxation by generalized landau damping, Phys. Rev. Lett. 68 (18) (1992) 2730-2733.

[14] D. Sagan, On the physics of landau damping, American Journal of Physics 62 (5) (1994) 450-462. URL http://link.aip.org/link/?AJP/62/450/1

[15] R. Bilato, M. Brambilla, The role of collisions in the quasilinear theory of radio-frequency heating and current drive in nearly collisionless plasmas, Plasma Physics and Controlled Fusion 46 (9) (2004) $1455-1465$.

URL http://stacks.iop.org/0741-3335/46/1455

[16] B. V. Chirikov, A universal instability of many-dimensional oscillator systems, Physics Reports 52 (1979) 263-379.

[17] A. J. Lichtenberg, M. A. Lieberman, Regular and Chaotic Dynamics, Springer, 1992.

[18] I. D. Kaganovich, Effects of collisions and particle trapping on collisionless heating, Phys. Rev. Lett. 82 (2) (1999) 327-330.

[19] R. W. Gould, T. M. O’Neil, J. H. Malmberg, Plasma wave echo, Phys. Rev. Lett. 19 (5) (1967) $219-222$ 